\title{
The equatorial meandering of abyssal ocean currents
}

\author{
G. E. Swaters \\ Applied Mathematics Institute, Department of Mathematical \& Statistical \\ Sciences and Institute for Geophysical Research, University of Alberta, \\ Canada
}

\begin{abstract}
Numerical simulations of the nonlinear primitive equations show that meridionally-flowing western-boundary grounded abyssal ocean currents, which are in a planetary-geostrophic dynamical balance in midlatitudes, become increasingly nonlinear and turn eastward as they approach the tropics developing into a meandering stationary planetary wave structure as they flow along the equator. A distinguished steady-state nonlinear dynamical asymptotic theory is introduced into the equatorial $\beta$-plane shallow water equations that describes the nonlinear stationary equatorial wave structure.
\end{abstract}

\section{Introduction}

In the Labrador and Norwegian/Greenland seas water associated with northward flowing surface ocean currents can become sufficiently dense due to sporadically intense atmospheric cooling that they sink to the bottom and start to flow back to the equator as deep western boundary currents (DWBC). This process starts the northern hemispheric contribution to the thermohaline circulation, or the convective overturning of the world's ocean, which is the dominant way that incoming solar heating is distributed deep into the ocean and is ultimately responsible for much of the temporal variability in Earth's climate.

Away from the equator, the motion of these deep or abyssal water masses is governed by a so-called nonlinear planetary-geostrophic dynamical balance $[1,2]$ in which the flows are essentially geostrophically-balanced gravity-driven density currents on a sloping bottom in a differentially rotating fluid. As these currents approach the equator, the geostrophic dynamics necessarily breaks down since the 
Coriolis parameter goes to zero but the pressure gradient remains nonzero. Thus, the theoretical question arises concerning the dynamical balances that govern the equatorial dynamics of these abyssal flows. The oceanographic data clearly shows that these flows cross the equator and circulate around the entire the globe [3].

Numerical simulations of the nonlinear primitive equations $[3,4]$ show that as these flows approach the equator they accelerate, turn eastward and the thickness of the abyssal water mass diminishes. These properties can all be understood as a consequence of the breakdown of geostrophy at the equator [1,2]. However, the numerical simulations $[3,4]$ further show that once the current turns eastward along the equator a stationary nonlinear planetary wave structure develops in which the current oscillates about the equator as it flows toward the eastern side of the ocean basin. The principal purpose of the present contribution is to present a preliminary description for the dynamics responsible for this steady-state nonlinear equatorial wave-like structure. A complete midlatitude-equatorial theory is presented in [5].

\section{Derivation of the equatorial model}

We work with a standard two-layer reduced-gravity equatorial $\beta$-plane model that assumes that the density in the upper layer is equal to a constant $\rho_{1}$ and the density in the lower layer is given by a constant $\rho_{2}$ with stable stratification (i.e., $\rho_{2}>\rho_{1}$ ). The upper layer is assumed to be infinitely deep and motionless. Although it is possible to work in spherical coordinates [1], for convenience, we work in Cartesian coordinates.

The steady-state reduced-gravity inviscid shallow water equations for an equatorial $\beta$-plane are given by

$$
\begin{gathered}
u u_{x}+v u_{y}-\beta y v=-g^{\prime}\left(h+h_{B}\right)_{x}, \\
u v_{x}+v v_{y}+\beta y u=-g^{\prime} h_{y}, \\
(h u)_{x}+(h v)_{y}=0,
\end{gathered}
$$

in which subscripts denote partial derivatives (unless otherwise noted), $x$ is the eastward coordinate, $y$ is the northward coordinate, $u(x, y)$ and $v(x, y)$ are the eastward and northward velocities, respectively, $p=\rho_{2} g^{\prime}\left(h+h_{B}\right)$ is the dynamic pressure, $g^{\prime} \equiv\left(\rho_{2}-\rho_{1}\right) g / \rho_{2}>0$ is the reduced gravity, $\beta=2 \Omega / R$ where $\Omega=2 \pi$ rads/day is Earth's angular frequency and $R \simeq 6400 \mathrm{~km}$ is the radius of the Earth, $h(x, y)>0$ is the height of the abyssal current above the zonally-varying topography whose height above some reference depth is given by $h_{B}=h_{B}(x)$.

Equations (1), (2) and (3) are solved assuming that at $y=y_{0}>0$ the flow is geostrophically-balanced and that

$$
h\left(x, y_{0}\right)=h_{0}(x) \equiv\left\{\begin{array}{c}
H_{*}\left(1-x^{2} / a^{2}\right) \text { when }|x| \leq a, \\
0 \text { when }|x|>a
\end{array}\right.
$$


where $H_{*}$ is the maximum height of the abyssal current located at the point $x=0$ and $a>0$ is the abyssal current half-width located along $y=y_{0}$. The abyssal height (4) contains two groundings located at $x= \pm a$, respectively.

The bottom topography in our analysis is the parabolic profile given by

$$
h_{B}=\gamma\left(x_{0}-x\right)^{2},
$$

where $x_{0}>a$ is the zonal location of the point of maximum depth located to the east of the downslope grounding associated with $h_{0}(x)$, and $\gamma>0$ is a parameter that is chosen so that the average value of the topographic slope immediately under $h_{0}(x)$ is reasonably consistent with observations of the underlying bottom slope associated with the DWBC [2] in midlatitudes, i.e.,

$$
\frac{1}{2 a} \int_{-a}^{a} h_{B}^{\prime}(x) d x=-s<0 \Longrightarrow \gamma=s /\left(2 x_{0}\right),
$$

where $s>0$ is the slope parameter.

In midlatitudes the appropriate nondimensionalization scheme is given by $[1,5]$

$$
\left.\begin{array}{c}
x=L \widetilde{x},\left(y, y_{0}\right)=R\left(\widetilde{y}, \widetilde{y}_{0}\right), v=V \widetilde{v}, u=(V L / R) \widetilde{u} \\
\left(h, h_{B}, h_{0}\right)=\left(2 \Omega V L / g^{\prime}\right)\left(\widetilde{h}, \widetilde{h}_{B}, \widetilde{h}_{0}\right), p=2 \Omega V L \rho_{2} \widetilde{p},
\end{array}\right\}
$$

where $L=a$ is the zonal length scale and $V=V_{\text {nof }} \equiv s g^{\prime} /(2 \Omega)$ is the meridional velocity scale where $V_{\text {nof }}$ is the Nof speed [6].

Substitution of (7) into (1)-(5) yields the nondimensional equations, after dropping the tildes, given by

$$
\begin{gathered}
\varepsilon \alpha^{2}\left(u \partial_{x}+v \partial_{y}\right) u-y v=-\left(h+h_{B}\right)_{x}, \\
\varepsilon\left(u \partial_{x}+v \partial_{y}\right) v+y u=-h_{y}, \\
(u h)_{x}+(v h)_{y}=0,
\end{gathered}
$$

where the reduced pressure is $p=h+h_{B}$, and where

$$
\begin{gathered}
h\left(x, y_{0}\right)=h_{0}(x) \equiv\left\{\begin{array}{c}
H\left(1-x^{2}\right) \text { when }|x| \leq 1, \\
0 \text { when }|x|>1,
\end{array}\right. \\
h_{B}(x)=\frac{1}{2 \delta}(1-\delta x)^{2} \Longrightarrow h_{B}^{\prime}(x)=-1+\delta x,
\end{gathered}
$$

with

$$
\varepsilon \equiv \frac{g^{\prime} s}{4 a \Omega^{2}}, \alpha \equiv \frac{a}{R}, H \equiv \frac{H_{*}}{s a}, \delta \equiv \frac{a}{x_{0}},
$$

where $\varepsilon$ is the Rossby number, $\alpha$ is the aspect ratio between the zonal and meridional length scales, $H$ is the nondimensional maximum height of $h_{0}(x)$, $\delta^{-1}$ is the nondimensional position of the point of maximum depth. 
Typical values for the parameters appropriate for the DWBC are [2]

$$
g^{\prime} \simeq 10^{-3} \mathrm{~m} / \mathrm{s}^{2}, s \simeq 5.6 \times 10^{-3}, a \simeq 100 \mathrm{~km},
$$

which implies that the meridional and zonal velocity scalings are, respectively,

$$
V_{\text {nof }} \simeq 3.9 \mathrm{~cm} / \mathrm{s} \text { and } V_{\text {nof }} L / R \simeq 0.06 \mathrm{~cm} / \mathrm{s}
$$

suggesting that

$$
\varepsilon \simeq 2.6 \times 10^{-3}, \alpha \simeq 1.6 \times 10^{-2} \Longrightarrow O\left(\varepsilon^{2}\right) \lesssim \alpha^{2} \lesssim O(\varepsilon)
$$

The abyssal height scale would be

$$
s a \simeq 560 \mathrm{~m} \Longrightarrow H \simeq O(1),
$$

and assuming that (at least) $x_{0} \simeq 1000 \mathrm{~km}$, it follows that

$$
O(\varepsilon)<\delta=10^{-1}<1
$$

We assume the Rossby number $\varepsilon$ as the fundamental underlying asymptotic parameter.

The leading order (as $\varepsilon \rightarrow 0$ ) solution to (8)-(10), which satisfies (11) is given by the nonlinear planetary-geostrophic flow $[1,5]$

$$
(u, v)=\frac{1}{y}\left(-\partial_{y}, \partial_{x}\right)\left(h+h_{B}\right),
$$

where $h(x, y)$ is obtained from

$$
\begin{gathered}
h(x, y)=\frac{y}{y_{0}} h_{0}(\tau), \\
h_{B}(\tau)+\left(\frac{y_{0}-y}{y_{0}}\right) h_{0}(\tau)=h_{B}(x) .
\end{gathered}
$$

From our perspective, the most important properties associated with the midlatitude solution (14)-(16) is that $h \rightarrow 0^{+}$and $u, v \rightarrow-\infty$ while maintaining finite volume fluxes $u h, v h$ as $y \rightarrow 0$. This behavior, which is a consequence of the breakdown of geostrophy, suggests the flow is accelerating and becoming "more" nonlinear as the equator is approached. As it turns out [5], the flow transitions through two inertial boundary layers as it enters the equatorial region. 
The first of these inertial boundary layers is described by substituting the "intermediate" (tilde) equatorial variables

$$
u=\varepsilon^{-1 / 3} \widetilde{u}, v=\varepsilon^{-1 / 3} \widetilde{v}, h=\varepsilon^{1 / 3} \widetilde{h}, y=\varepsilon^{1 / 3} \xi,
$$

into (8)-(10), yielding

$$
\begin{gathered}
\alpha^{2}\left(\varepsilon^{1 / 3} \widetilde{u} \partial_{x}+\widetilde{v} \partial_{\xi}\right) \widetilde{u}-\xi \widetilde{v}=-\left(h_{B}+\varepsilon^{1 / 3} \widetilde{h}\right)_{x}, \\
\varepsilon^{1 / 3} \widetilde{u} \widetilde{v}_{x}+\widetilde{v} \widetilde{v}_{\xi}+\xi \widetilde{u}=-\widetilde{h}_{\xi}, \\
\varepsilon^{1 / 3}(\widetilde{u} \widetilde{h})_{x}+(\widetilde{v})_{\xi}=0 .
\end{gathered}
$$

Within the context $\alpha^{2} \lesssim O(\varepsilon)$, it follows that the leading order problem (as $\varepsilon \rightarrow 0$ ) is given by

$$
\begin{gathered}
\xi \widetilde{v}=h_{B}^{\prime}(x), \\
\widetilde{v} \widetilde{v}_{\xi}+\xi \widetilde{u}=-\widetilde{h}_{\xi}, \\
(\widetilde{v} \tilde{h})_{\xi}=0,
\end{gathered}
$$

which must be solved subject to the matching conditions

$$
\widetilde{h} \rightarrow \frac{\xi h_{0}(\widetilde{\tau}(x))}{y_{0}}, \widetilde{u} \rightarrow-\frac{h_{0}(\widetilde{\tau}(x))}{\xi y_{0}}, \widetilde{v} \rightarrow \frac{h_{B}^{\prime}(x)}{\xi},
$$

as $\xi \rightarrow \infty$ where $\widetilde{\tau}(x)=\tau(x, 0)$.

The solution to (21)-(23) is given by

$$
\begin{gathered}
\widetilde{h}(x, \xi)=\frac{\xi h_{0}(\widetilde{\tau}(x))}{y_{0}}, \widetilde{v}(x, \xi)=\frac{h_{B}^{\prime}(x)}{\xi}, \\
\widetilde{u}(x, \xi)=\frac{1}{\xi}\left[\frac{\left(h_{B}^{\prime}(x)\right)^{2}}{\xi^{3}}-\frac{h_{0}(\widetilde{\tau}(x))}{y_{0}}\right] .
\end{gathered}
$$

The most important things to observe in the solution (25) and (26) is that $\widetilde{v}(x, \xi)$ is no longer coupled to $\widetilde{h}$ but remains singular as $\xi \rightarrow 0^{+}$. The intermediate solution has accomplished the physically important task of turn the flow eastward since $\widetilde{u}>0$ as $\xi \rightarrow 0$. However, $\widetilde{u}$ remains singular as $\xi \rightarrow 0$. This suggests the emergence of yet another "inner" inertial boundary layer. 
The second of these inertial boundary layers is described by substituting the "inner" (careted) equatorial variables

$$
\widetilde{u}=\alpha^{-4 / 3} \widehat{u}, \widetilde{v}=\alpha^{-1 / 3} \widehat{v}, \widetilde{h}=\alpha^{1 / 3} \widehat{h}, \xi=\alpha^{1 / 3} \zeta,
$$

into (18)-(20), yielding

$$
\begin{gathered}
\nu \widehat{u u_{x}+} \widehat{v u}_{\zeta}-\zeta \widehat{v}=-\left(h_{B}+(\alpha \varepsilon)^{1 / 3} \widehat{h}\right)_{x}, \\
\nu \widehat{u v}_{x}+\widehat{v v_{\zeta}}+\zeta \widehat{u}=-\alpha \widehat{h}_{\zeta}, \\
\nu(\widehat{u} \widehat{h})_{x}+(\widehat{v} \widehat{h})_{\zeta}=0,
\end{gathered}
$$

where $\nu \equiv\left(\varepsilon / \alpha^{2}\right)^{1 / 3} \simeq 2.21 \simeq O(1)$.

Within the context $\alpha \lesssim O(\sqrt{\varepsilon})$, it follows that the leading order problem (as $\varepsilon \rightarrow 0$ ) associated with (28)-(30) will be the quasi-linear hyperbolic system of equations

$$
\begin{gathered}
\left(\nu \widehat{u} \partial_{x}+\widehat{v} \partial_{\zeta}\right) \widehat{u}-\zeta \widehat{v}=-h_{B}^{\prime}(x), \\
\left(\nu \widehat{u} \partial_{x}+\widehat{v} \partial_{\zeta}\right) \widehat{v}+\zeta \widehat{u}=0, \\
\nu(\widehat{u} \widehat{h})_{x}+(\widehat{v} \widehat{h})_{\zeta}=0,
\end{gathered}
$$

which must be solved subject to the matching conditions

$$
\widehat{h} \rightarrow \frac{\zeta h_{0}(\widetilde{\tau}(x))}{y_{0}}, \widehat{u} \rightarrow \frac{\left(h_{B}^{\prime}(x)\right)^{2}}{\zeta^{4}}, \widehat{v} \rightarrow \frac{h_{B}^{\prime}(x)}{\zeta}
$$

as $\zeta \rightarrow \infty$. Importantly, observe that the determination of $(\widehat{u}, \widehat{v})$ is no longer coupled to the determination of $\widehat{h}$.

\section{The equatorial planetary wave structure}

To solve for the velocity field in the inner equatorial region we use the method of characteristics. The characteristic equations are given by

$$
\begin{gathered}
\frac{d x}{d \sigma}=\nu \widehat{u}, \\
\frac{d \zeta}{d \sigma}=\widehat{v}, \\
\frac{d \widehat{u}}{d \sigma}=\zeta \widehat{v}-h_{B}^{\prime}(x), \\
\frac{d \widehat{v}}{d \sigma}=-\zeta \widehat{u},
\end{gathered}
$$


subject to the boundary conditions

$$
\begin{gathered}
\left.x\right|_{\sigma=0}=\mu,\left.\zeta\right|_{\sigma=0}=\zeta_{0} \equiv \frac{\varepsilon^{1 / 3}+(\beta \varepsilon)^{1 / 3}}{2(\beta \varepsilon)^{1 / 3}}=2.5 \\
\left.\widehat{u}\right|_{\sigma=0}=u_{0}(\mu) \equiv \frac{\left(h_{B}^{\prime}(\mu)\right)^{2}}{\zeta_{0}^{4}},\left.\widehat{v}\right|_{\sigma=0}=v_{0}(\mu) \equiv \frac{h_{B}^{\prime}(\mu)}{\zeta_{0}},
\end{gathered}
$$

where $\mu$ is the coordinate across the characteristics and gives a parametric representation of the boundary data along $\zeta=\zeta_{0}$ and $\sigma$ is the coordinate along the characteristics. In order to numerically compute the characteristics it is necessary to "start" the solution at a finite value of $\zeta$. The choice of $\zeta_{0}$ corresponds, formally, to a value that is "outside" the inner equatorial region but "inside" the intermediate equatorial region.

Even with $h_{B}^{\prime}(x)$ given by (12) there is no known analytical solution to the characteristic equations (except in the case where $h_{B}^{\prime}(x) \equiv 0$ when a solution can be written in terms of elliptic functions [7]). Thus, the characteristic equations must be solved numerically $[4,5]$. The numerical solutions show the emergence of a meridionally narrow stationary planetary wave structure along the equator $[4,5]$. If the abyssal flow ultimately completely crosses the equator and continues to flow southward on the eastern side of the basin along the topographic slope, the characteristics must necessarily intersect in the inner equatorial region and it is at these intersection regions that dissipation must be introduced to remove the formation of discontinuities in the solution [5]. Physically, dissipation is necessary in order for the required potential vorticity adjustment to occur allowing crossequatorial flow [3-5] of a midlatitude planetary-geostrophic current.

The essential details of the planetary wave structure can be revealed with the following approximation. The emergence of a meridionally narrow stationary planetary wave structure along the equator in the numerical solution of the characteristic equations $[4,5]$ suggests that, as a first approximation, one can neglect the $\zeta \widehat{v}$ term in (37), which allows (35) and (37) to be written in the form

$$
\frac{d^{2} \widehat{u}}{d \sigma^{2}}+\delta \nu \widehat{u}=0
$$

which is solved subject to

$$
\left.\widehat{u}\right|_{\sigma=0}=u_{0}(\mu) \text { and }\left.\frac{d \widehat{u}}{d \sigma}\right|_{\sigma=0}=1-\delta \mu,
$$

the solution of which is given by

$$
\widehat{u}(\mu, \sigma)=\phi(\mu) \cos [\sqrt{\delta \nu} \sigma-\theta(\mu)]
$$

where

$$
\phi \equiv \sqrt{u_{0}^{2}(\mu)+\frac{(1-\delta \mu)^{2}}{\delta \nu}}, \theta \equiv \arctan \left(\frac{1-\delta \mu}{u_{0}(\mu) \sqrt{\delta \nu}}\right)
$$


It follows from (37) (having neglecting the $\zeta \widehat{v}$ term) that

$$
x(\mu, \sigma)=[1+\phi \sqrt{\delta \nu} \sin (\sqrt{\delta \nu} \sigma-\theta)] / \delta,
$$

which can be "inverted" to yield

$$
\sigma=\widehat{\sigma}(x) \equiv \frac{1}{\sqrt{\delta \nu}}\left[\theta+\arcsin \left(\frac{\delta x-1}{\phi \sqrt{\delta \nu}}\right)\right],
$$

which determines how $\sigma$ is a function of $x$ along each characteristic $\mu=$ constant. The $x$-coordinate "starts" at $x=\mu$ and continues eastward, first moving down the topography and then through the position of maximum depth located at $x=1 / \delta$, and then up the eastern topographic slope until it reaches its maximum eastern point located at $x=x_{\max } \equiv(1+\phi \sqrt{\delta \nu}) / \delta$ (which occurs for $\sigma$ given by $\sqrt{\delta \nu} \sigma-\theta=\pi / 2$ where $\widehat{u}=0)$.

If (36) and (43) are substituted into (38) one obtains the Mathieu equation

$$
\frac{d^{2} \zeta}{d \sigma^{2}}+\phi \cos (\sqrt{\delta \nu} \sigma-\theta) \zeta=0
$$

which must be solved subject to

$$
\left.\zeta\right|_{\sigma=0}=\zeta_{0},\left.\frac{d \zeta}{d \sigma}\right|_{\sigma=0}=v_{0}
$$

While it is possible to write the solution to (47) and (48) in terms of the Mathieu sine and cosine functions [5] and then substitute in (46) to obtain $\zeta=$ $\zeta(x)$ along each characteristic and thereby numerically determine the equatorial wave structure, a simpler explicit representation can be obtained by replacing $\cos (\sqrt{\delta \nu} \sigma-\theta)$ in (47) with its average positive value over the interval $0 \leq$ $\sqrt{\delta \nu} \sigma-\theta \leq \pi / 2$, which is given by $2 / \pi$ [4], i.e., we replace (47) with

$$
\frac{d^{2} \zeta}{d \sigma^{2}}+\frac{2 \phi}{\pi} \zeta=0
$$

the solution of, which satisfies (48), is given by

$$
\zeta=\varphi(\mu) \cos [\sqrt{2 \phi(\mu) / \pi} \sigma-\psi(\mu)],
$$

where

$$
\varphi(\mu) \equiv \sqrt{\zeta_{0}^{2}+\pi v_{0}^{2} /(2 \phi)}, \psi(\mu) \equiv \arctan \left(\frac{v_{0}}{\zeta_{0} \sqrt{2 \phi / \pi}}\right) .
$$

Finally, if (46) is substituted into (50) we have, along each characteristic, that

$$
\zeta=\varphi \cos \left\{\left[\sqrt{2 \phi /(\delta \nu \pi)}\left[\theta+\arcsin \left(\frac{\delta x-1}{\phi \sqrt{\delta \nu}}\right)\right]-\psi\right]\right\} .
$$


The solution (52) describes a stationary planetary wave with amplitude $\varphi$ with a "wavelength" over the first cycle, denoted as $\lambda$, given by

$$
\lambda=\frac{1+(\delta \mu-1) \cos \left(\sqrt{\frac{2 \nu \delta \pi^{3}}{\phi}}\right)+u_{0} \sqrt{\delta \nu} \sin \left(\sqrt{\frac{2 \nu \delta \pi^{3}}{\phi}}\right)}{\delta} .
$$

As an example, the wavelength associated with the $\mu=0$ characteristic would be $\left.\lambda\right|_{\mu=0} \simeq 18.3$, which dimensionally corresponds to a zonal wavelength of about $1830 \mathrm{~km}$. The maximum meridional deflection of this wave is $\varphi \simeq 2.52$, which dimensionally corresponds to about $550 \mathrm{~km}$. These estimates are consistent with the numerical simulations $[3,4]$.

\section{Summary}

An asymptotic theory has been advanced to describe the stationary planetary equatorial wave that is observed in numerical simulations of the equatorial crossing of grounded abyssal currents. Our calculations produce estimates for the meridional deflection and zonal wavelength that are consistent with the numerical simulations. Further work is, however, required to determine the role that baroclinicity, horizontal and bottom friction, and diapycnal mixing has on the structure of this equatorial wave.

\section{Acknowledgement}

Preparation of this paper was partially supported by the Natural Sciences and Engineering Research Council of Canada.

\section{References}

[1] Swaters, G. E., Flow of grounded abyssal ocean currents along zonallyvarying topography on a rotating sphere. Geophysical and Astrophysical Fluid Dynamics, 107, pp. 564-586, 2013.

[2] Swaters, G. E., On the meridional flow of source-driven abyssal currents in a stratified basin with topography. Part II. Numerical Simulation. Journal of Physical Oceanography, 36, pp. 356-375, 2006.

[3] Nof, D. \& Borisov, S., Inter-hemispheric oceanic exchange. Quarterly Journal of the Royal Meteorological Society, 124, pp. 2829-2866, 1998.

[4] Kim, A., Swaters, G. E. \& Sutherland, B. R., Cross-equatorial flow of grounded abyssal ocean currents. Submitted to Geophysical and Astrophysical Fluid Dynamics, 2014.

[5] Swaters, G. E., The steady midlatitude-equatorial dynamics of a grounded deep-western boundary current in a meridional basin with parabolic bottom topography. Submitted to Journal of Fluid Mechanics, 2014.

[6] Nof, D., The translation of isolated cold eddies on a sloping bottom. Deep-Sea Research 30, pp. 171-182, 1983.

[7] Cushman-Roisin, B., Motion of a free particle on a beta-plane. Geophysical and Astrophysical Fluid Dynamics, 22, pp. 85-102, 1982. 